\title{
Nanomagnetics with lasers
}

\author{
R C BUDHANI* and RAJIB K RAKSHIT \\ Department of Physics, Indian Institute of Technology Kanpur, Kanpur 208 016, India
}

\begin{abstract}
Both liquid and vapour phase pulsed laser deposition (PLD) techniques have been used to synthesize nanophase magnetic alloys of CoPt. While the liquid route results in soft phase (disordered fcc) nanoparticles of CoPt near equiatomic composition dispersed in a surfactant-polymer matrix, the conventional vapour phase PLD allows growth of high coercivity nanoscale structures of CoPt on (001) $\mathrm{SrTiO}_{3}$. The magnetization, $M(T)$, dynamics of the colloidal particles is examined. Two distinct particle distributions are established from analysis of $M(T)$ data, in conformity with results of electron microscopy. In vapour deposited films at low growth rate $(\sim 0.4 \AA / s)$, morphology changes from a self-similar fractal to nanodots as the deposition temperature is raised from $700-800^{\circ} \mathrm{C}$. The large lattice mismatch between $(001) \mathrm{SrTiO}_{3}$ and the $a c / b c$ plane of $L 1_{0}$ ordered phase imparts tensile strain to the films whose morphological manifestations can be suppressed at high growth rates.
\end{abstract}

Keywords. Nanomagnetics; lasers.

\section{Introduction}

Magnetic materials in zero, one and two-dimensional geometries such as clusters consisting of a few thousand atoms (Sun et al 2000; Rakshit and Budhani 2006), wires with nanometer scale diameter (Tatara and Fakuyama 1997; Hong and Giordano 1998) and ultra thin films, respectively (Shima et al 2004; Rakshit et al 2006, 2008), have been at the centre stage of condensed matter/ materials physics, and are the basis of a myriad of technologies. The fundamental interest in low dimensional magnetic materials emanates from the fact that a macroscopic property such as magnetization, which is a consequence of the cooperative response of millions of spins, can be altered in a non-trivial manner if a large fraction of these spins are made to reside on the surfaces or interfaces of a magnetic entity (Kodama 1999). In most of the magnetic systems of technological relevance, the moment comes from the spin of the electron. The technology of controlling the motion of electrons in solids has been mastered to perfection since the invention of transistor half a century ago. This has been the basis of all electronic devices of mind boggling complexity and performance characteristics, and yet, only half of the potential of electron has been exploited. Tapping the spin degree of freedom of electrons is the challenge of the present century. This is the field of nanomagnetism. A preview of its fascinating aspects, both scientific and technological, is presented by effects such as giant magnetoresistance (GMR) (Hartman 2000; Hirota et al 2002), tunneling magnetoresistance (TMR) (Miyazaki and Tezuka 1995;

*Author for correspondence (rcb@iitk.ac.in)
Moodera et al 1995; Parkin 2002), and the phenomena of spin blockade (Takahashi and Maekawa 1998; Ciorga et al 2002), spin coherence (Wolf et al 2001; Žutić et al 2004) and spin decoherence (Žutić et al 2004). These effects hold great potential for fabrication of nonvolatile random access memories and ultrafast computation using the concepts of quantum mechanics.

In order to understand, utilize and enjoy the scientific challenges posed by these effects, one must have the tools to synthesize nanoscale magnetic structures reproducibly and economically. A major part of the research being conducted in the Condensed Matter - Low Dimensional Systems Laboratory of the Physics Department at IIT Kanpur is geared towards these objectives (Rakshit and Budhani 2006; Rakshit et al 2006, 2008). Both liquid and vapour phase synthesis routes are being used to prepare colloidal solution of nanoparticles, quantum dot structures supported on substrates and ultra thin $(\sim 10 \mathrm{~nm})$ single crystal films. High power pulsed lasers are the key tools used in these synthesis routes. In the following, we will describe how the enormous power $(\sim 10$ million Watts/ pulse) available from a pulsed laser is used for these purposes.

\section{Results and discussion}

\subsection{Pulsed laser ablation in aqueous medium (PLAAM): Colloidal solutions of hard magnetic alloys}

Several chemical methods have been developed in recent years to synthesize monodispersed colloids of ferromagnetic nanoparticles. While these techniques work well for simple elemental and oxide-based ferromagnets, there are 
difficulties in producing alloy nanoparticles through the chemical route as the process involves toxic and often inflammable metallorganics combined with difficulties in stoichiometry control. We have developed a pulsed NdYAG laser based technique to synthesize nanoparticles of multicomponent alloys. The colloidal solutions of nanoparticles prepared in this manner are promising for drug delivery, ferrofluid mechanics, conducting inks and other futuristic technologies. A sketch of this technique is shown in figure 1 along with a photograph of the facility in figure 2 . We have successfully synthesized nanoparticles

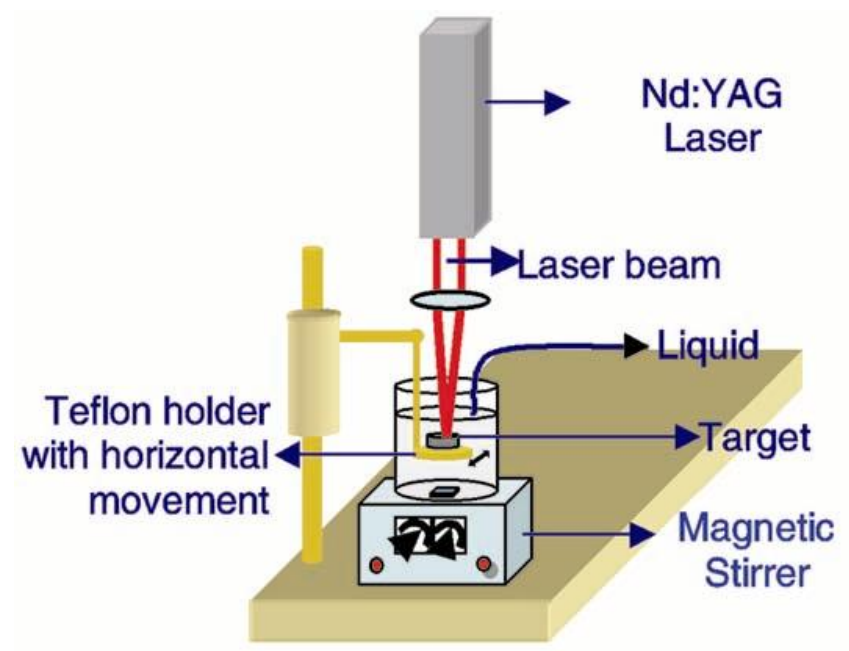

Figure 1. A schematic of the PLAAM process.

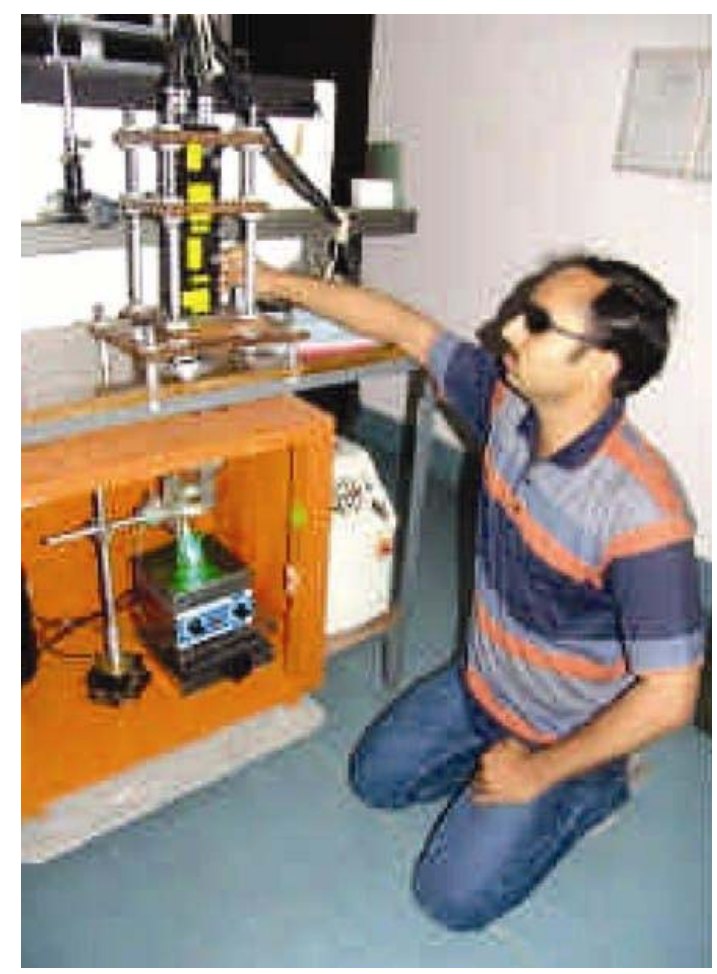

Figure 2. This picture depicts the PLAAM facility. of $\mathrm{Ag}, \mathrm{Cr}, \mathrm{YBa}_{2} \mathrm{Cu}_{3} \mathrm{O}_{7}$ and most importantly of $\mathrm{CoPt}$, a very promising alloy for high density magnetic data storage.

For the case of $\mathrm{CoPt}$ nanoparticles, a plate of $\mathrm{CoPt}$ alloy synthesized by arc melting of high purity cobalt and platinum chunks followed by homogenization at high temperature was suspended horizontally in a bath of triply distilled water. A focused beam of pulsed Nd-YAG laser (Quantel Big Sky, pulse width, $\sim 6$ nanoseconds) irradiated the plate from the top. The laser was operated at $20 \mathrm{~Hz}$ with a combined energy density of $14 \mathrm{~J} / \mathrm{cm}^{2}$ at $1064 \mathrm{~nm}$ and $532 \mathrm{~nm}$ wavelengths. A light brown but clear solution, with no evidence of any sedimentation, was obtained after $25 \mathrm{~min}$ of ablation. In order to avoid agglomeration of particles, sodium dodecylsulfate (SDS) was added to the solution immediately after the ablation process and homogenized by ultrasonification for $10 \mathrm{~min}$. The colloidal solution was concentrated through the process of vacuum evaporation of excess water. The larger particles and agglomerates have been separated subsequently through centrifugation at $\sim 3000 \mathrm{~g}(4000 \mathrm{rpm})$ of the resultant solution for $15 \mathrm{~min}$.

The morphology and composition of the CoPt nanoparticles were characterized using transmission electron microscopy (TEM) (JEOL JEM-3010, $300 \mathrm{kV}$ ) and energy dispersive X-ray (EDX) spectroscopy, respectively. Samples for these measurements were prepared by drying a drop of the colloidal solution on a suitable template. Commercial carbon coated $\mathrm{Cu}$ grids and highly ordered pyrolytic graphite (HOPG) plates were used as templates for TEM and EDX measurements, respectively. It was evident from TEM micrographs that the nanoparticles had a droplet type of morphology and their size varied from $\sim 1.5$ to $\sim 6 \mathrm{~nm}$. A point-to-point EDX analysis of the

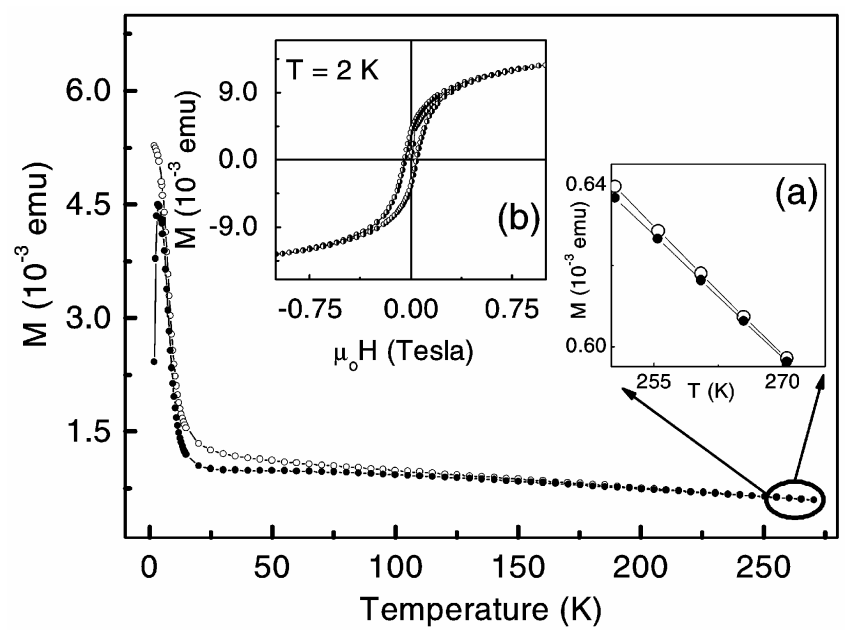

Figure 3. Temperature dependence of the zero-field-cooled (solid circles) and field-cooled (open circles) magnetization of the CoPt nanoparticles dispersed in a polymer matrix measured at 500 Gauss. Magnified view in the temperature window of $250 \mathrm{~K}$ to $275 \mathrm{~K}$ is shown in the inset (a). Inset (b) shows the hysteretic behaviour of magnetization vs field at $2 \mathrm{~K}$. 
vacuum dried nanoparticles of $\mathrm{CoPt}$ revealed a constant $\mathrm{Co} / \mathrm{Pt}$ ratio. Solid samples for magnetization measurements were prepared by adding a low melting point $(\sim 330 \mathrm{~K})$ water soluble analytical grade polymer (polyethylene glycol) to the colloid and dried completely through vacuum evaporation. This process also minimizes interparticle magnetic interactions and help in the formation of micelles by shielding the repulsion between the ionic heads of the surfactant.

We now focus on the magnetic response of SDSencapsulated $\mathrm{CoPt}$ alloy nanoparticles dispersed in the polymeric matrix. In order to check the contribution of the surfactant polymer matrix to the total magnetization, CoPt-free samples of SDS-PEG were measured. The response of this matrix is predominantly diamagnetic which becomes significant only at fields in excess of $\sim 0.5$ Tesla. The field-cooled (FC) and zero-field-cooled (ZFC) magnetization measured at 500 Gauss as a function of temperature is shown in figure 3 . These low-field $\mathrm{ZFC}$ and FC curves, which first bifurcate at $T \leq 260 \mathrm{~K}$, remain separated down to $\sim 10 \mathrm{~K}$ where the moment shows a rapid increase on lowering the temperature. A magnified view of low-field ZFC and FC curves in the temperature window of $250 \mathrm{~K}$ to $275 \mathrm{~K}$ is shown in the inset 'a' for clarity. The value of the FC and ZFC moment remains the same over a narrow interval of temperature, and then the ZFC moment shows a sharp drop below $4 \mathrm{~K}$. Measurements of magnetization as a function of field below $2 \mathrm{~K}$ also show a distinct hysteresis (inset ' $b$ ' of figure 3 ). The separation of the zero-field-cooled and field-cooled branches of magnetization curves on decreasing the temperature is a characteristic feature of an agglomeration of superparamagnetic particles. Two distinct particle distributions are suggested by the behaviour of the ZFC and FC magnetization curves which bifurcate twice on cooling below room temperature. Our electron microscopy results show these distribution peaks at $1.3 \mathrm{~nm}$ and $5.9 \mathrm{~nm}$ (Rakshit and Budhani 2006).

A further verification of the size distribution comes from the magnetization relaxation measurements on our nanoparticles. Since the relaxation rate of magnetic moment in nanoparticles is a sharp function of the particle size, distribution of the latter in our case is expected to manifest itself in the characteristic relaxation time. The Néel expression for the relaxation time of a superparamagnetic particle is

$$
\tau=\tau_{0} \exp \left(\Delta E(B) / k_{\mathrm{B}} T\right)
$$

where $\tau_{0}$ is the characteristic relaxation time, $k_{\mathrm{B}}$ the Boltzmann constant. In our case, the relaxation measurement was performed by cooling the sample down to the measurement temperature in 2 Tesla applied field. Once the temperature was stabilized, the field was reduced to zero in 'no overshoot' mode in $300 \mathrm{~s}$. After a waiting time of $100 \mathrm{~s}$, the remanent magnetization was measured as a function of time. In figure 4 , we have shown the decay of magnetic moment with time for CoPt alloy nanoparticles cooled in an applied field of 2 Tesla to the measurement temperature of $2 \mathrm{~K}$. The field-cooled (FC) and zerofield-cooled (ZFC) magnetization measured at 2 Tesla as a function of temperature is shown in the inset. As can be seen in figure 4 and in the inset of figure 4 , there is an order of magnitude drop in magnetic moment when the first data point of the magnetic relaxation has been taken. This is basically due to the relaxation of smaller particles. Therefore, the relaxation data presented in the figure reflect only the distribution of characteristics relaxation time for bigger particles with certain size distribution. A fit to the relaxation data using the simple first order exponential decay function is shown as a solid line in figure 4. Clearly, this dependence does not explain the relaxation adequately. The inexplicability of this fitting is due to the presence of particle size distribution in our sample as stated before. At $10 \mathrm{~K}$, the nanoparticles were found to relax almost instantly on removal of the field. The magnetization, therefore, appears to remain constant during the accessible time of measurement. This is typical of a paramagnetic system or a SPM system above $T_{\mathrm{C}}$, where the particles react immediately to a field change. At $2 \mathrm{~K}$, however, most of the particles are blocked and a typical activated relaxation is observed which follows the Arrhenius law.

In order to ascertain the magnitude of anisotropy in this system, we have recorded the temperature dependent magnetization at a higher field ( 2 Tesla). If the energy associated with the applied field exceeds the anisotropy energy, then one would expect a overlapping set of ZFC

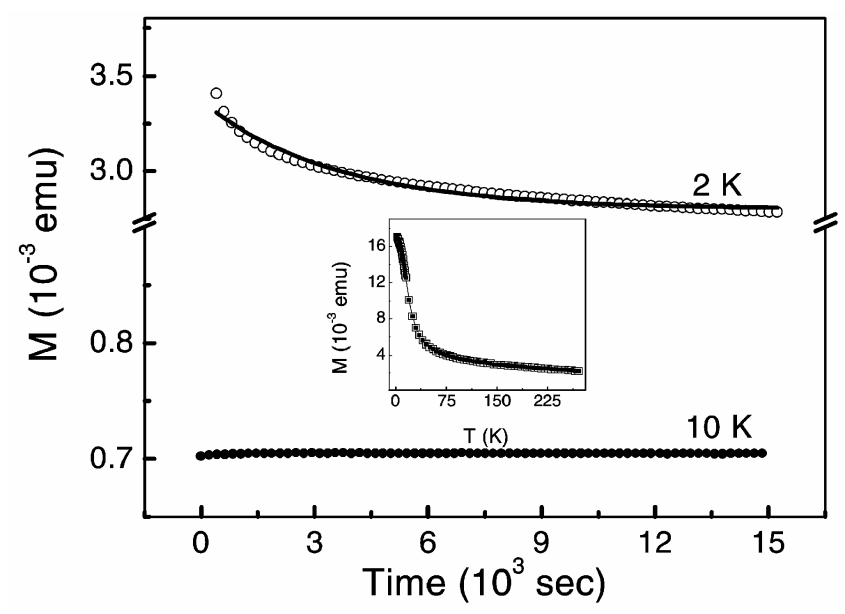

Figure 4. Open circles show the result of magnetic relaxation measurement at $2 \mathrm{~K}$ on a sample after cooling it to $2 \mathrm{~K}$ in the presence of 2 Tesla field. The inadequacy in fitting the experimental data with single exponential decay (solid line) is clear from the figure. Solid circles show the magnetic relaxation at $10 \mathrm{~K}$ under the same field cooled condition. Inset of the figure shows temperature dependence of the zero-field-cooled (solid squares) and field-cooled (open squares) magnetization of the CoPt nanoparticles measured at 2 Tesla field. 

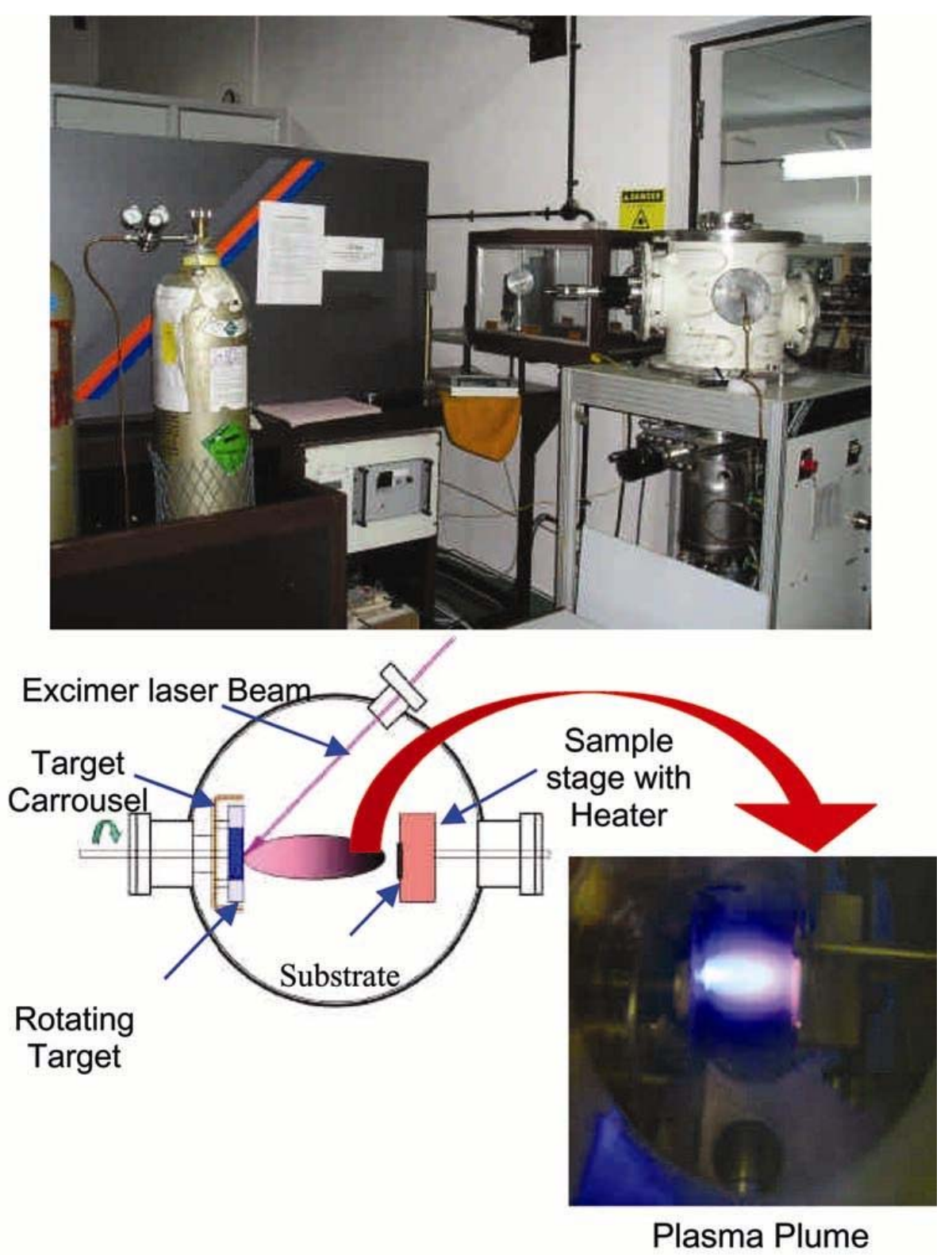

Figure 5. Vapour phase pulsed laser deposition.

and FC curves, since at all temperatures the preferential magnetization orientation of the nanoparticles will be essentially the direction of the field. Only a weak paramagnetic response is expected as a function of temperature, reminiscent of the thermal fluctuation of individual moments about the direction of applied field. The result of this high-field measurement is shown in the inset of figure 4 . As expected, we find no separation between ZFC and FC curves within the experimental limits. Further details of analysis of the magnetization data are given elsewhere (Rakshit and Budhani 2006).
2.2 Vapour phase condensation of laser ablated plume on single crystal surfaces: Preparation of quantum dots of hard magnetic materials

Pulsed laser ablation is a powerful tool to synthesize thin films of multicomponent alloys and compounds because of its ability to maintain the stoichiometry of the target material in the condensed thin film. A schematic view of pulsed laser deposition (PLD) process is shown in figure 5. Here a pellet of the multicomponent material such as $\mathrm{Co}_{50} \mathrm{Pt}_{50}$ alloy to be deposited as thin film is fired upon with ultra-short pulses ( $20 \mathrm{~ns})$ of radiation from a UV 
laser. Because of the stupendous power density of the laser pulse, small chunks of target simply explode and the vapour thus generated accelerates in the forward direction with supersonic speed. Since the process occurs so rapidly that all components of the target reach the substrate in spite of their differing vapour pressures.

The energy carried by laser ablated atoms is $\sim 100$ times more than the energy of atoms evaporated from a simple thermal source. This excess energy plays a key role in the stabilization of novel non-equilibrium phases. The physics and materials science of condensation of the vapours emanating from the target onto a single crystal substrate offer many challenges. After landing on the substrate, the atoms arrange themselves, starting from step edges to form a continuous single crystal layer. The surface, interface and elastic energies of this layer change during the deposition process and the atomic arrangement on the surface evolves so as to minimize the sum of these ener-
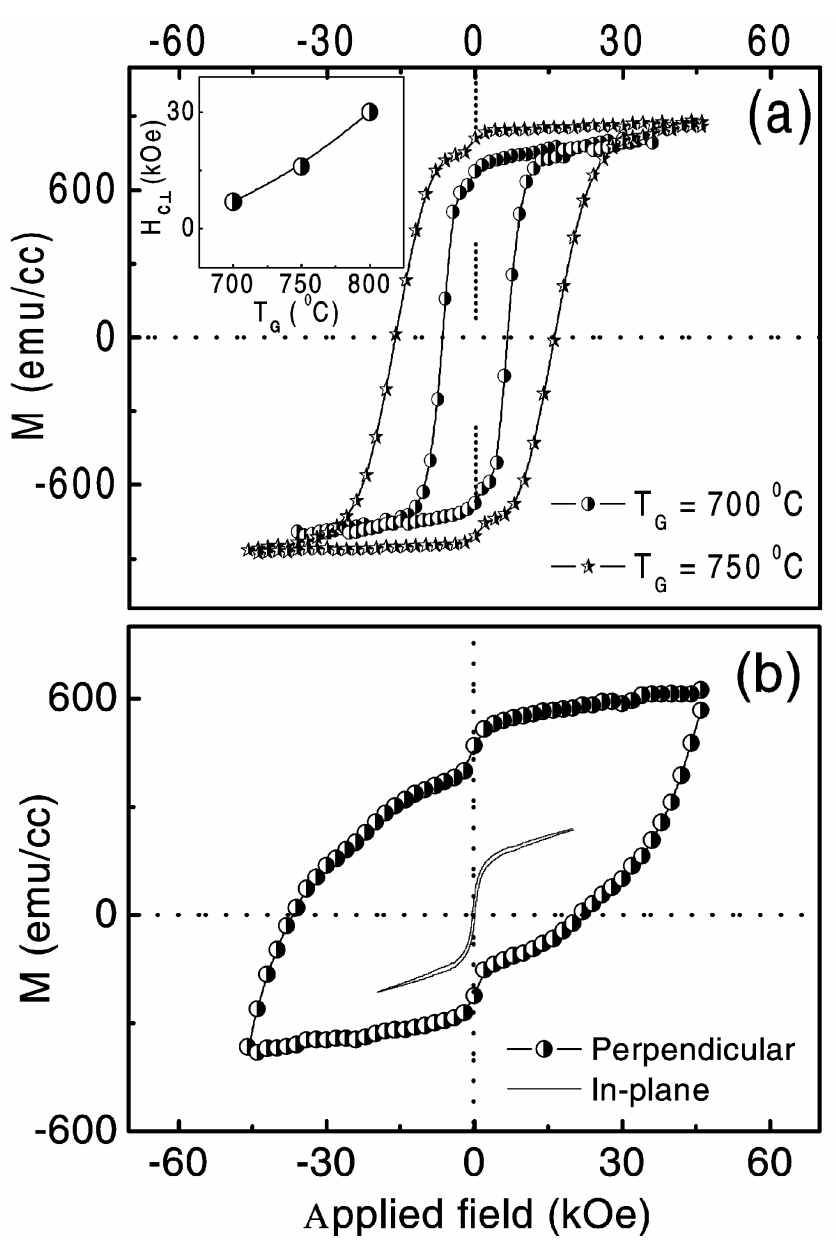

Figure 6. (a) Magnetization curves of CoPt films deposited at $700^{\circ} \mathrm{C}$ and $750^{\circ} \mathrm{C}$. The magnetic field was applied along the film normal. (b) Perpendicular as well as in-plane magnetization curve for the CoPt film deposited at $800^{\circ} \mathrm{C}$. Inset of (a) shows the evolution of $H_{\mathrm{c} \perp}$ with $T_{\mathrm{G}}$. Solid line in the figure is guide to the eye. gies. The elastic strain energy of the film grows quadratically with film thickness, and if the bulk lattice parameter of the structure that is being deposited is even a few percent different from that of the substrate, nanometer size islands can form on the surface to minimize the total energy.

Studies of magnetization reversal in thin films of $\mathrm{FePt}$ and $\mathrm{CoPt}$ are technologically important due to their large magnetic coercivity in the face-centred-tetragonal $(f c t)$ $L 1_{0}$ phase. Most of the earlier research employed sputtering or molecular beam epitaxy (MBE) to grow textured polycrystalline thin films of CoPt and FePt. Majority of these works have been carried out on $\mathrm{MgO}$ (001) single crystal substrate. The lattice parameter of $\mathrm{MgO}$ (cubic, $a=4.21 \AA$ ) is larger by $8.5 \%$ and $9.6 \%$ as compared to the basal plane unit cell parameter of $L 1_{0} \mathrm{FePt}$ and CoPt, respectively. In order to minimize the effects of such large lattice mismatch on epitaxial growth, graded interfaces consisting of seed layers have been used.

The (001) face of $\mathrm{SrTiO}_{3}$ [STO] (cubic, $a=3.905 \AA$ ) provides an ideal square planar lattice for epitaxial growth of the $L 1_{0}$ phase of CoPt whose lattice parameters in the bulk form are $a=b=3.803 \AA$ and $c=3.701 \AA$. Here we report the use of pulsed laser deposition (PLD) to fabricate $c$-axis oriented epitaxial $L 1_{0} \mathrm{CoPt}$ films which display a coercive field as high as $\approx 30 \mathrm{kOe}$ at ambient temperature.

A KrF excimer laser $(\lambda=248 \mathrm{~nm}$, pulse width, $\sim 20 \mathrm{~ns})$ was used to ablate CoPt alloy target in an all metal seal PLD chamber maintained at 50 mTorr pressure of ultra high purity $(99.9999 \%) \mathrm{N}_{2}$ buffer, which enhanced removal of particulate matter from the ablation plume. Films were deposited on (001) STO wafers at 700, 750 and $800^{\circ} \mathrm{C}$ with a growth rate $\left(G_{\mathrm{r}}\right)$ ranging from $0.4 \AA / \mathrm{s}$ to $1 \AA / \mathrm{s}$, and then annealed in situ for $25 \mathrm{~min}$ after the growth. While the laser frequency was kept fixed at $10 \mathrm{~Hz}$, energy density on the target was increased from $2.5 \mathrm{~J} / \mathrm{cm}^{2}$ to $3.6 \mathrm{~J} / \mathrm{cm}^{2}$ to achieve a higher $G_{\mathrm{r}}$. Under these conditions of growth, the film thickness uniformity was limited to $10 \times 10 \mathrm{~mm}^{2}$. This inherent drawback of PLD can be overcome to some extent by translating the substrate during growth.

Films of thickness, $\approx 500 \AA$, calibrated using a surface profilometer, were synthesized and their crystallographic structure probed using X-ray diffraction (XRD) in the standard $\theta-2 \theta$ geometry. The surface topography of the films was investigated by scanning electron microscopy (SEM). For magnetization measurements, we have used a superconducting quantum interference device based magnetometer.

The out-of-plane magnetic field $\left(H_{\perp}\right)$ dependence of magnetization $\left(M\left(H_{\perp}\right)\right)$ of the films deposited at several $T_{\mathrm{G}}$ is shown in figure 6 . These data reveal that while the $M\left(H_{\perp}\right)$ of the films with $T_{\mathrm{G}} \leq 750^{\circ} \mathrm{C}$ saturates at $H_{\perp}<30 \mathrm{kOe}$, for the sample deposited at $800^{\circ} \mathrm{C}$ the saturation field was beyond the maximum d.c.-field (46 kOe) 

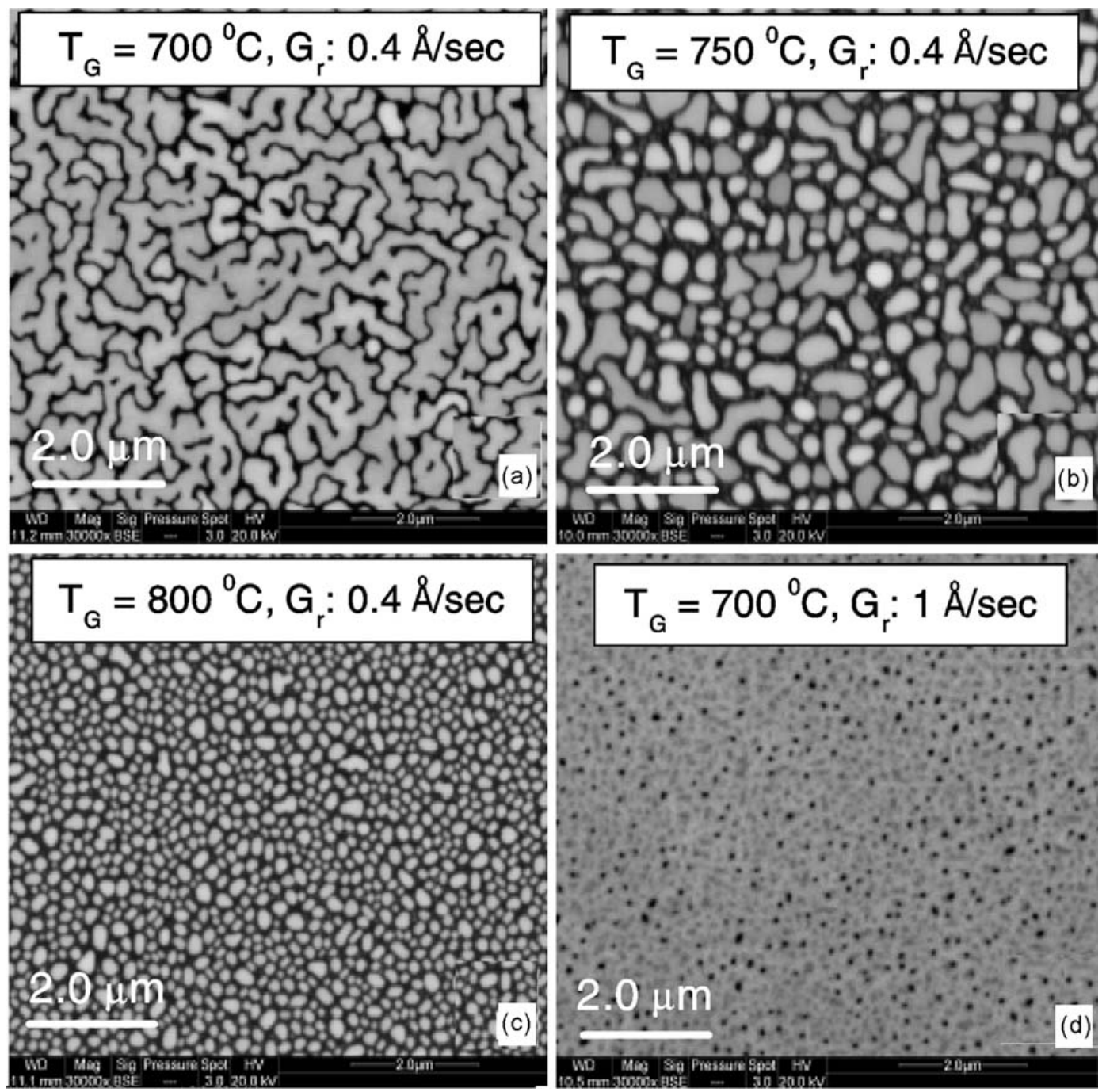

Figure 7. SEM images of $500 \AA \mathrm{CoPt}$ thin films deposited with different deposition conditions.

used in these measurements. This is indicated by the hysteresis loop shown in figure 6(b) which is neither symmetrical nor centred about the origin, and has no reversible region even at the maximum applied field. In figure $6(\mathrm{~b})$, the $M-H$ loop for the in-plane field $\left(H_{\|}\right)$is also plotted. A very thin hysteresis with coercivity $H_{\mathrm{c} \|} \approx 400$ Oe confirms the X-ray diffraction result that there is no $c$-axis variant present in films of $T_{\mathrm{G}}=800^{\circ} \mathrm{C}$. The uniaxial magnetic anisotropy, $K_{\mathrm{u}}$, for this film determined from $M\left(H_{\perp}\right)$ and $M\left(H_{\|}\right)$data, comes out to be $4.62 \times 10^{6} \mathrm{~J} / \mathrm{m}^{3}$, which is very close to the maximum value $\left(\sim 5 \times 10^{6} \mathrm{~J} / \mathrm{m}^{3}\right)$ reported in the literature. The dependence of the coercive field, $H_{\mathrm{c} \perp}$, on the growth temperature is plotted in the inset of figure 6(a). A very large $H_{\mathrm{c} \perp}$ of $\approx 30 \mathrm{kOe}$ is seen in the films deposited at $800^{\circ} \mathrm{C}$. Since the $M-H$ loop is asymmetric, the $H_{\mathrm{c} \perp}$ quoted here is an average of the forward and reverse fields at which the $M$ goes to zero. It is important to mention here that the limited field range ( $\approx 46 \mathrm{kOe}$ ) of the SQUID magnetometer used here did not permit full saturation of the $M-H$ loop. Thus, value of $H_{\mathrm{c} \perp}$ and $K_{\mathrm{u}}$ listed here may be lower than the actual value.

The magnetic behaviour of these films is intimately linked with their fascinating topography which evolves due to a non-zero epitaxial strain (Rakshit et al 2006, 2007). As seen in figure $7(\mathrm{a})$, the film deposited at $700^{\circ} \mathrm{C}$ with a lower $G_{\mathrm{r}}$ is a self-similar fractal. At $T_{\mathrm{G}}=750^{\circ} \mathrm{C}$ [figure 7(b)], however, the maze-like pattern breaks up into islands of arbitrary shape. With further increase in $T_{\mathrm{G}}$, the islands become nearly circular with a mean size of $\approx 200 \mathrm{~nm}$ [figure 7(c)]. The demagnetization of such isolated and presumably single domain particles will lead to a large coercivity if their uniaxial anisotropy energy is large. The large average size of the fractals in figure 7(a) grown at lower temperature $\left(T_{\mathrm{G}}=700^{\circ} \mathrm{C}\right)$ is not likely to support a mono-domain structure. The demagnetization then proceeds via the motion of weakly pinned domain walls with a consequently lower coercivity. The evolution of morphology with $T_{\mathrm{G}}$ and $G_{\mathrm{r}}$ is intimately linked with the epitaxial strain, $\varepsilon$, defined as 


$$
\varepsilon=\left[\left(d_{\text {bulk }}-d_{\text {substrate }}\right) / d_{\text {bulk }}\right] \times 100,
$$

where $d$ is lattice parameter. The $\varepsilon$ for in-plane and outof-plane $c$-axis growth of the $f c t$ phase on STO is $-5.5 \%$ and $-2.7 \%$, respectively, and for the $f c c$ phase, $\varepsilon=-1.7 \%$. From this analysis it is evident that the $c$-axis in-plane films are under a large tensile strain $(\varepsilon=-5 \cdot 5 \%)$ which is partially relieved at lower $G_{\mathrm{r}}$ via formation of the fractal structure (figure 7(a)). The higher $G_{\mathrm{r}}$ seems to suppress this stress relief mechanism due to kinetic considerations and a smooth morphology emerges (figure 7(d)). At higher $T_{\mathrm{G}}$, thermodynamics assists the growth of the less strained $c$-axis out-of-plane variant $(\varepsilon=-2 \cdot 7 \%)$. However, $\varepsilon=$ $-2.7 \%$ is still large enough to cause a Stranski-Krastanov or Volmer-Weber type growth of quantum dots (figure 7(c)).

\section{Conclusions}

In summary, pulsed laser ablation technique has been used to create nanophase magnetic alloys both in colloidal form and as self-assembled thin films. The CoPt nanodots synthesized on $\mathrm{SrTiO}_{3}$ substrates show extremely large coercivity $\left(H_{\mathrm{c} \perp} \approx 3\right.$ Tesla) at ambient temperature. Magnetization relaxation data are consistent with super-hard magnetic character of the nanodots.

\section{Acknowledgement}

This research has been supported under the Nano Science Initiative of the Department of Science \& Technology, Government of India.

\section{References}

Ciorga M, Pioro-Ladriere M, Zawadzki P, Hawrylak P and Sachrajda A S 2002 Appl. Phys. Lett. 802177

Hartman U (ed.) 2000 Magnetic multilayers and giant magnetoresistance (Berlin: Springer)

Hirota E, Sakakima H and Inomata K 2002 Giant magnetoresistance devices (Berlin: Springer)

Hong K and Giordano N 1998 J. Phys.: Condens. Matter 10 L401

Kodama R H 1999 J. Magn. Magn. Mater. 200359

Miyazaki T and Tezuka N 1995 J. Magn. Magn. Mater. 139 L231

Moodera J S, Kinder L R, Wong T M and Meservey R 1995 Phys. Rev. Lett. 743273

Parkin S S P 2002 Spin dependent transport in magnetic nanostructures (eds) S Maekawa and T Shinjo (New York: Taylor and Francis) pp. 237-271

Rakshit R K and Budhani R C 2006 J. Phys. D: Appl. Phys. 39 1743

Rakshit R K, Bose S K, Sharma R and Budhani R C 2006 Appl. Phys. Lett. 89202511

Rakshit R K, Bose S K, Sharma R, Budhani R C, Vijaykumar T, Neena S J and Kulkarni G U 2008 J. Appl. Phys. 103 023915

Shima T, Takanashi K, Takanashi Y K and Hono K 2004 Appl. Phys. Lett. 852571

Sun S, Murray C B, Weller D, Folks L and Moser A 2000 Science 2871989

Takahashi S and Maekawa S 1998 Phys. Rev. Lett. 801758

Tatara G and Fukuyama H 1997 Phys. Rev. Lett. 783773

Wolf S A, Awschalom D D, Buhrman R A, Daughton J M, von Molnár S, Roukes M L, Chtchelkanova A Y and Treger D M 2001 Science 2941488

Žutić I, Fabian J and Sharma S D 2004 Rev. Mod. Phys. 76323 